\title{
Monolithic PDMS Laminates for Dielectric Elastomer Transducers through Open-Air PlasmATreatment
}

\author{
Hassouneh, Suzan Sager; Oubæk, Jakob; Daugaard, Anders Egede; Skov, Anne Ladegaard
}

Published in:

Polymer-Plastics Technology and Materials

Link to article, DOI:

$10.1080 / 03602559.2015 .1098702$

Publication date:

2016

Document Version

Peer reviewed version

Link back to DTU Orbit

Citation (APA):

Hassouneh, S. S., Oubæk, J., Daugaard, A. E., \& Skov, A. L. (2016). Monolithic PDMS Laminates for Dielectric Elastomer Transducers through Open-Air PlasmATreatment. Polymer-Plastics Technology and Materials, 55(8), 769-774. https://doi.org/10.1080/03602559.2015.1098702

\section{General rights}

Copyright and moral rights for the publications made accessible in the public portal are retained by the authors and/or other copyright owners and it is a condition of accessing publications that users recognise and abide by the legal requirements associated with these rights.

- Users may download and print one copy of any publication from the public portal for the purpose of private study or research.

- You may not further distribute the material or use it for any profit-making activity or commercial gain

- You may freely distribute the URL identifying the publication in the public portal 


\section{Polymer-Plastics Technology and Engineering}

\section{Monolithic PDMS Laminates for Dielectric Elastomer Transducers through Open-Air PlasmA- Treatment}

\section{Suzan Sager Hassouneh, Jakob Oubæk, Anders Egede Daugaard \& Anne Ladegaard Skov}

To cite this article: Suzan Sager Hassouneh, Jakob Oubæk, Anders Egede Daugaard \& Anne Ladegaard Skov (2015): Monolithic PDMS Laminates for Dielectric Elastomer Transducers through Open-Air PlasmA-Treatment, Polymer-Plastics Technology and Engineering, DOI: 10.1080/03602559.2015.1098702

To link to this article: http://dx.doi.org/10.1080/03602559.2015.1098702

View supplementary material 주

Accepted author version posted online: 05 Nov 2015.

Submit your article to this journal $\widetilde{T}$

Q View related articles $\llbracket$

View Crossmark data $\nearrow$ 


\title{
Monolithic PDMS Laminates for Dielectric Elastomer Transducers Through Open- Air Plasma-Treatment
}

Suzan Sager Hassouneh ${ }^{1}$, Jakob Oubæk ${ }^{2}$, Anders Egede Daugaard ${ }^{1}$, Anne Ladegaard Skov $^{1}$

${ }^{1}$ The Danish Polymer Centre, Department of Chemical and Biochemical Engineering, Technical University of Denmark, Lyngby, Denmark, ${ }^{2}$ Danfoss Polypower, Nordborg, E-mail:al@kt.dtu.dk

\begin{abstract}
The present study investigates the use of an open-air plasma-treatment system for the surface modification of polydimethylsiloxane (PDMS), in order to improve layer-to-layer adhesion. The procedure presented herein is more cost efficient compared to conventional vacuum-based plasma-treatment, and it is performed at different speeds and distances away from the nozzle, to investigate how these two parameters influence the resulting interfacial layer of two fully cured PDMS films. The plasma-treatment is determined not to alter mechanical properties compared to the single film, while peel forces are sufficient to avoid delamination during operation.
\end{abstract}

KEYWORDS: PDMS, Monolithic, Plasma, Open-air

\section{INTRODUCTION}

In recent years, interest in smart materials has increased. Smart materials respond to external stimuli such as temperature, $\mathrm{pH}$ and electrical or magnetic fields by changing, for example, their size and shape. Dielectric elastomers are classed as smart materials, and they can be used for actuation, sensing and energy harvesting ${ }^{[1,2]}$. Dielectric 
elastomer transducers are thin elastomeric films coated with compliant electrodes. When a voltage is applied across the elastomer, it deforms due to electrostatic forces between the electrodes, meaning that the film becomes thinner and expands in area ${ }^{[2,3]}$. This principle can be utilised for a range of different applications and in a variety of configurations such as tubes, rolls and diaphragms. Transducers are generally multilayer systems, prepared from acrylic, urethane or PDMS elastomers. A dielectric elastomer film has a given energy density, and by adding multiple layers to an element, the energy density of the element increases, since the supporting structure is relatively heavy. Furthermore, multiple layers make the element more robust, thus prolonging the lifetime of the transducer.

Several multilayer configurations have been investigated previously, in an attempt to improve the performance of transducers as well as to control the direction of actuation. These include stacked, helical and folded configurations ${ }^{[4-8]}$.

A stacked actuator consists of multiple layers connected mechanically in series and in parallel electrically. This configuration requires multiple production steps due to structural discontinuity ${ }^{[7,8]}$, and the layers are mechanically independent of each other, which causes friction between the layers and subsequently reduces their lifespan. In addition, air can be trapped between the layers, which can cause a decline in electrical breakdown strength. The helical actuator is composed of two helices, one coated with compliant electrodes and the other smeared with uncured dielectric elastomer. The two helices are coupled so that electrodes and elastomer alternate, and these coupled helices 
are placed in a mould containing uncured dielectric elastomer and the element is kept in this mould for 12 hours at room temperature until the dielectric elastomer has cured. The dielectric elastomer acts as an insulating material to prevent air discharge during actuation, and the actuator has a monolithic structure ${ }^{[5]}$. A folded actuator consists of a continuous strip of elastomer coated with compliant electrodes, folded and sealed with a thin coating of identical elastomer material. The folded and helical structures are fabricated to a thickness of between 0.5 and $0.8 \mathrm{~mm}$ for each layer. This relatively bulky thickness, as well as the thin coating used as insulation, means that these structures require higher working voltages and results in an increase in mechanical resistance ${ }^{[5,6]}$.

One way to eliminate the above mentioned issues is to adhere the layers together without the use of additional elastomer, which will make the transducer airtight between layers and eliminate any friction between the adjacent layers. Furthermore, it will prevent the use of additional insulating material that causes an increase in mechanical resistance and subsequently an increase in working voltages.

Adhering multiple layers together is commonly used in the fabrication of microfluidic devices and microelectromechanical systems (MEMS), and different approaches have been investigated for these systems ${ }^{[9,10]}$. One approach for silicone dielectric elastomers is to use a sticky layer, containing a deficient amount of crosslinker, which enables direct bonding to another surface with excess crosslinker on the surface ${ }^{[11]}$. The disadvantage of this method is that the layers have different mechanical and electrical properties, which subsequently will influence the overall performance of the transducer. This method is 
suitably only for silicone elastomers based on the silylation reaction such as the elastomers from Madsen et $\mathrm{al}^{[12-13]}$. A second approach involves adding uncured PDMS or crosslinker as glue between the layers ${ }^{[14-16]}$, which increases the thickness of the dielectric material and in turn reduces thickness strain, according to the actuation equation derived by Pelrine et al ${ }^{[17]}$. Again this method relies on specific chemistries. A third approach is to plasma-treat the PDMS films. In this approach $\mathrm{Si}-\mathrm{CH}_{3}$ groups on the surface of the silicone elastomer - no matter the crosslinking chemistry-are modified to silanol groups $(\mathrm{Si}-\mathrm{OH})$, and when films are brought together covalent siloxane bonds (Si$\mathrm{O}-\mathrm{Si}$ ) are formed in the interface ${ }^{[9]}$. A variety of gases, such as argon, carbondioxide, oxygen and air, can be used to create plasma ${ }^{[18,19]}$, and for all gases, plasma is produced in a low-vacuum environment, which is a prohibitively expensive step in an industrial process. Detailed studies of oxygen-treated PDMS films can be found in the studies of Bhattacharya et $\mathrm{al}^{[20]}$ and Millare et $\mathrm{al}^{[21]}$. An alternative way to plasma-treat would be to use an open-air plasma-treatment system, the advantage of which would be the elimination of the low-vacuum environment as well as the possibility of a continuous industrial process, which will ultimately decrease processing costs.

This study investigates the possibilities of using an open-air plasma-treatment for PDMS films, in order to make monolithic structures. The focus in this study is to determine the optimal parameters required to perform plasma-treatment, such as speed and distance to the nozzle, by investigating the mechanical and electrical properties of the monolithic layers, as well as peel force, to determine if the laminates will detach during operation. 


\section{EXPERIMENTAL}

\section{Materials And Methods}

Elastosil LR3043/30 was purchased from Wacker Chemie AG, Germany. Elastosil LR3043/30 is a two-component system whereby component A contains vinyl-terminated polydimethylsiloxane (PDMS) and a platinum catalyst, and component B contains PDMS crosslinker and vinyl-terminated PDMS amongst others. The utilized carrier web is from Danfoss PolyPower and consists of a surface-treated high-temperature resistant acrylate based resin.

The plasma-treatment is performed using Plasmatreat RD1004 with nozzle head 22892 both acquired from Plasmatreat GmbH, Germany. This nozzle has a working diameter of $55 \mathrm{~mm}$.

The samples are placed on a lift table, so that the distance to the nozzle can be adjusted. The lift table is also attached to a moving unit that regulates the speed at which the plasma-treater moves across the sample.

Rheological characterisation is performed on a TA Instruments AR2000 rheometer with parallel plate geometry and a diameter of $25 \mathrm{~mm}$ and a controlled strain of $2 \%$.

Measurements are performed in the frequency range $100-0.01 \mathrm{~Hz}$ and with a normal force of 6-8 N. A laminated sample is compared with a single film to investigate if plasmatreatment influences mechanical properties, while rheological characterisation is 
performed for a single film and laminate with thicknesses of $0.5 \mathrm{~mm}$ and $1 \mathrm{~mm}$, respectively.

An in-house-built device, based on international standards (IEC 60243-1 (1998) and IEC 60243-2 (2001)), is used to measure electrical breakdown strength, while sample thickness is calculated by weighing. The sample is placed between two spherical electrodes and the distance is set to the sample thickness on a digital indicator with 0.001 $\mathrm{mm}$ resolution. A stepwise increase in voltage (50-100 V/step) at a rate of $0.5-1$ steps/s is applied at the point of contact ${ }^{[22]}$, and electrical breakdown strength is measured as the voltage at which the elastomer breaks down and short-circuits. Electrical breakdown strength is measured at 12 points for each sample, and an average of the measurements is then indicated as the breakdown strength of the sample.

For peel measurements, a Zwick/Roell zmart.pro material tester is used. The sample (130 $\mathrm{mm} * 25 \mathrm{~mm}$ ) is attached to a metal plate. The laminate is separated at one end at a distance of approximately $25 \mathrm{~mm}$ and the free end is clamped to a gripper. The metal plate with the attached sample is then fixed to a second gripper, before the device moves the gripper up and the force used to peel the films from one another is measured. The laminate is separated at a $180^{\circ}$ angle and at a separation rate of $152 \mathrm{~mm} / \mathrm{min}$.

\section{Procedure}

The elastomer (LR3043/30) is coated as a thin layer on a carrier web and is subsequently cured in an oven ${ }^{[23][19]}$. The elastomer is kept on the carrier web, in order to achieve a 
smooth surface without wrinkles and to ease handling. Samples of $20 \mathrm{~cm}$ by $20 \mathrm{~cm}$ are prepared at distances away from the nozzle ranging from $5 \mathrm{~mm}$ to $15 \mathrm{~mm}$ in $5 \mathrm{~mm}$ increments and at speed intervals of $5 \mathrm{~m} / \mathrm{min}$ to $20 \mathrm{~m} / \mathrm{min}$ in $5 \mathrm{~m} / \mathrm{min}$ increments.

\section{RESULTS AND DISCUSSION}

Samples are prepared by plasma-treating the surface of LR3043/30 PDMS films, which are adhered together by contacting the treated sides together, thereby resulting in the formation of the laminate interface shown in Figure 1.

As shown in Figure 1 the plasma-treatment is performed at different distances between the sample and the nozzle and at different speeds across the films. To determine the influence of plasma- treatment on the surface of the elastomer films, scanning electron micrographs (SEM) using an energy-dispersive X-ray detector (EDS) were recorded for both treated and non-treated films (supplementary information). The investigation showed no obvious changes between the pristine PDMS surface and the plasma-treated surfaces. Therefore, it was concluded that any differences in bonding strength between the surfaces would be the result of surface changes on a molecular level.

\section{Mechanical Properties}

Rheological measurements are performed to investigate the mechanical properties of a laminate and then to determine if the plasma-treated interfacial layer has changed the overall mechanical properties of the film. In particular, it is important to determine if the laminates have higher interfacial stiffness, since stiffness in the interface will cause a 
reduction in actuation or cause disruption to the interface upon actuation. Mechanical properties of a plasma-treated sample are compared with a single non-plasma-treated film. The storage modulus is proportional to the crosslinking density, i.e. it indicates at a given frequency the amount of crosslinks, both dynamic (entanglements) and static (true covalent crosslinks $^{[23,24]}$. The loss modulus is an indicator of the viscous losses within the material. The loss modulus is commonly expressed as the loss tangent, $\tan \delta$, which is the ratio of storage to loss modulus. The storage moduli and loss tangents are depicted as functions of applied frequency at $23^{\circ} \mathrm{C}$ in Figure 2.

The storage modulus (G') shows a minor increase for the laminate compared to the single film. This minor difference can be ascribed to the difference in thicknesses of the samples, and it is not expected to be the result of a stronger interfacial bond. At low frequencies of the applied oscillation, the loss tangent is comparable with the single film, whereas at frequencies between the 1 and $10 \mathrm{~Hz}$ the laminate has a lower loss than the single film. This can be explained by a reduction of surface area per volume ${ }^{[25]}$, which confirms the monolithic nature of the laminate.

\section{Electrical Breakdown Strength}

Electrical breakdown strength is measured for the laminate at the previously specified distances and speeds, to investigate if plasma-treatment influences breakdown strength. Furthermore, the measurements are performed on single films, where both plasma-treated and non-plasma-treated films are investigated. Breakdown strengths for the different samples are shown in Figure 3. 
Figure 3 shows that breakdown strength decreases after plasma-treatment for both single films and laminates for all distances and speeds. There are no obvious correlations between the decrease in breakdown strength and the distances and speeds at which the samples are treated. A moderate decrease in breakdown strength is observed for the plasma-treated single films compared to the non-treated single films, which is due to the formation of polar silanol groups on the surface during plasma-treatment. For the laminates, the decrease in breakdown strength is due to higher sample volume and thus the higher likelihood of encountering a defect in the test specimen. Furthermore, during breakdown measurement, when high voltage is applied across the film volumetric Joule heating is expected ${ }^{[26]}$. For thicker films the removal of the excessive heat is slower than for thinner films, which subsequently will decrease breakdown strength as conductivity increases in line with increased temperature ${ }^{[27]}$. Zakaria et al ${ }^{[27]}$ measured breakdown strength for samples as single films with various thicknesses as well as samples consisting of multiple layers and showed that breakdown strengths of thicker single films decreased by $43 \%$ and the multiple layer samples decreased by $31 \%$. In comparison, the plasma-treated films in this study decreased by only $6 \%$ in breakdown strength, which shows that the plasma-treatment of the interface conserves breakdown strength and provides a more homogeneous laminate, which in turn confirms the monolithic structure of the sample.

To analyse the reliability of the elastomer films, a Weibull analysis is performed on the averaged breakdown strengths for each plasma-treatment condition. The fitted data can 
be seen in Table 1. First of all, it can be seen that the initial films (single films) are very comparable and fully obeys Weibull behaviour. The plasma-treatment clearly reduces the electrical performance of the films but no matter the conditions of the treatment the electrical properties remain similar as the averaged breakdown strengths of the single, plasma-treated films possess the same $\beta$ parameter as the ensemble of single films. This could mean that the lowest dose is above the percolation threshold for getting some additional surface conductivity. The lamination is of high quality since the $\eta$ of the laminated samples remain more or less unaltered compared to the single, plasma-treated films. Additional interfacial defects such as air bubbles or dust would have caused premature break down ${ }^{[28-29]}$. It is, however, noticeable that the width of the distribution increases ( $\beta$ decreases) which must mean that the mechanical strength of the interfaces vary so strongly that it causes variation in the electrical properties. This is due to the electrical breakdown strength scaling with the Young's modulus and thus with the storage modulus ${ }^{[29]}$.

\section{Peel Forces}

The interfacial layer strength has previously been measured by several methods ${ }^{[20-21]}$ such as by determination of contact angles due to the plasma-treatment causing the hydrophobic methyl groups to react to become hydrophilic hydroxyl groups. Since our target is to laminate two very flexible films and to have these laminates performing as stretchable capacitors, we test our films by means of peeling studies, a commonly applied method for evaluation of adhesives ${ }^{[30]}$. The data is shown in Figure 4. 
Figure 4 illustrates that peel strengths for the samples performed at distances of 5 and 15 $\mathrm{mm}$ are generally lower than what is observed at a distance of $10 \mathrm{~mm}$ to the nozzle. In addition, a much higher scattering of data is observed at these distances, with varying application speeds. An explanation for the observed variation could be that the dosage is either too high or too low to provide a uniform treatment of the sample. If the distance is too short, the intensity of the plasma might so high that silica particles are formed at the interface, which will result in poorer interfacial adhesion as discussed in the work by Millare et $\mathrm{al}^{[21]}$. If the distance is too high, though, this will lead to the intensity of the treatment being too low and the surface will not be significantly modified, thereby resulting in an insignificant reaction on the interface. Plasma-treatments performed at a distance of $10 \mathrm{~mm}$ show small variations in the peel forces at different application speeds and generally provide the strongest interface. Both the variation and the strength of the interface indicate that this is the optimal distance away from the sample to the plasma nozzle, where a sufficient degree of modification of the film surfaces has taken place, thus ensuring good interfacial bonding.

\section{CONCLUSION}

The use of an open-air plasma-treatment system to adhere silicone dielectric elastomers together, in order to improve the overall performance of dielectric electroactive transducers, has been investigated. This method eliminates the need for additional dielectric material as interfacial glue for the preparation of monolithic elements. The plasma-treatment was performed at different speeds and distances away from the nozzle. The resulting mechanical properties, breakdown strength and interfacial strength of the 
laminates were investigated. Rheological measurements showed that laminates are slightly stiffer than single films, although losses are comparable, which confirms the monolithic structure of the laminates. The electrical breakdown strength of the laminates investigated in this study decreased by only $6 \%$ compared to single films, which is a significantly lower reduction than what is observed for non-treated samples, which confirms the monolithic nature of the laminates.

A nozzle distance of $10 \mathrm{~mm}$ was identified as the optimal distance at which to perform plasma- treatment, as could be seen through low variations in peel forces as well as good interfacial strength at different application speeds. The peel forces were furthermore sufficiently high to ensure that the laminates would maintain their structure during operation.

The obtained results show that open-air plasma-treatment is a suitable method for pretreating PDMS films before adhesion and that it results in high-strength laminates performing as monolithic elements.

\section{REFERENCES}

[1] Brochu, P.; Pei, Q. Advances in dielectric elastomers for actuators and artificial muscles. Macromol. Rapid Commun. 2010, 31, 10-36.

[2] Zhang, X.; Löwe, C.; Wissler, M.; Jähne, B.; Kovacs, G. Dielectric elastomers in actuator technology. Adv. Eng. Mater. 2005, 7, 361-367. 
[3] O'Halloran, A.; O’Malley, F.; McHugh, P. A review on dielectric elastomer actuators, technology, applications, and challenges. J. Appl. Phys. 2008, 104, 071101. [4] Kovacs, G.; Düring, L.; Michel, S.; Terrasi, G. Stacked dielectric elastomer actuator for tensile force transmission. Sensors Actuators A Phys. 2009, 155, 299-307. [5] Carpi, F.; Migliore, A.; Serra, G.; De Rossi, D. Helical dielectric elastomer actuators. Smart Mater. Struct. 2005, 14, 1-7.

[6] Carpi, F.; Salaris, C.; De Rossi, D., Folded dielectric elastomer actuators. Smart Mater. Struct. 2007, 16, S300-S305.

[7] Schlaak, H. F.; Jungmann, M.; Matysek, M.; Lotz, P. Novel multilayer electrostatic solid-state actuators with elastic dielectric. Proc. SPIE, 2005, 5759, 121-133. [8] Pelrine, R.; Kornbluh, R.; Joseph, J. Electrostriction of polymer dielectrics with compliant electrodes as a means of actuation. Sensors Actuators A Phys., 1998, 64, 7785.

[9] Eddings, M. A.; Johnson, M. A.; Gale, B. K. Determining the optimal PDMSPDMS bonding technique for microfluidic devices. J. Micromechanics Microengineering, 2008, 18, 067001.

[10] Penskiy, I.; Gerratt, A. P.; Bergbreiter, S. Friction, adhesion and wear properties of PDMS films on silicon sidewalls. J. Micromechanics Microengineering, 2011, 21, 105013.

[11] Unger, M. A.; Chou, H.-P.; Thorsen, T.; Scherer, A.; Quake, S. R. Monolithic microfabricated valves and pumps by multilayer soft lithography. Science, 2000, 288, $113-116$. 
[12] Madsen, F. B.; Yu, L.; Daugaard, A. E.; Hvilsted, S.; Skov, A. L. Silicone

elastomers with high dielectric permittivity and high dielectric breakdown srtength based on dipolar copolymers. Polymer. 2014, 55, 6212-6219.

[13] Madsen, F. B.; Yu, L.; Daugaard, A. E.; Hvilsted, S.; Skov, A. L. A new soft dielectric silicone elastomer matrix with high mechanical integrity and low losses. $\mathbf{2 0 1 5}$, $5,10254-10259$

[14] Yu, L.; Daugaard, A. E.; Skov, A. L. Adhesion between polydimethylsiloxane layers by crosslinking. Adv. Sci. Technol. 2012, 79, 47-52.

[15] Yu, L.; Skov, A. L. Monolithic growth of partly cured polydimethylsiloxane thin film layers. Polym. J. 2014, 46, 123-129.

[16] Satyanarayana, S.; Karnik, R. N.; Majumdar, A. Stamp-and-stick roomtemperature bonding technique for microdevices. J. Microelectromechanical Syst. 2005, $14,392-399$.

[17] Pelrine, R.; Kornbluh, R.; Pei, Q.; Joseph, J. High-speed electrically actuated elastomers with strain greater than 100\%. Science, 2000, 287, 836-839.

[18] Ginn, B. T.; Steinbock, O. Polymer surface modification using microwave-ovengenerated plasma. Langmuir, 2003, 19, 8117-8118.

[19] McDonald, J. C.; Whitesides, G. M. Poly(dimethylsiloxane) as a material for fabricating microfluidic devices. Acc. Chem. Res. 2002, 35, 491-499.

[20] Bhattacharya, S.; Datta, A.; Berg, J. M.; Gangopadhyay, S. Studies on surface wettability of poly(dimethyl) siloxane (PDMS) and glass under oxygen-plasma treatment and correlation with bond strength. J. Microelectromech. Syst. 2005, 14, 590-597. 
[21] Millare, B.; Thomas, M.; Ferreira, A.; Xu, H.; Holesinger, M.; Vullev, V. I. Dependence of the quality of adhesion between poly(dimethylsiloxane) and glass surfaces on the conditions of treatment with oxygen plasma. Langmuir, 2008, 24, 1321813224.

[22] Kiil, H.-E.; Benslimane, M. Scalable industrial manufacturing of DEAP. Proc. SPIE, 2009, 7287, 72870R-1-72870R-10.

[23] Larsen, A. L.; Hansen, K.; Sommer-Larsen, P.; Hassager, O.; Bach, A.; Ndoni, S.; Jørgensen, M. Elastic properties of nonstoichiometric reacted PDMS networks. Macromolecules, 2003, 36, 10063-10070.

[24] Jensen, M. K.; Hassager, O.; Rasmussen, H. K.; Skov, A.L.; Bach, A.; Koldbech, H. Planar elongationof soft polymeric networks. Rheol Acta, 2010, 49, 1-13.

[25] Hassouneh, S. S.; Daugaard, A. E.; Skov, A. L. Design of elastomer structure to facilitate incorporation of expanded graphite in silicones without compromising electromechanical integrity. Macromol. Mater. Eng. 2015, 300, 542-550.

[26] Zakaria, S.; Morshuis, P. H. F.; Mohamed, B.; Gernaey, K. V.; Skov, A.L. The electrical breakdown of thin dielectric elastomers $\square$ : thermal effects. Proc. SPIE, 2014, 9056, $90562 \mathrm{~V}$.

[27] Zakaria, S.; Morshuis, P. H. F.; Benslimane, M. Y.; Yu, L.; Skov, A. L. The electrical breakdown strength of prestretched elastomers with and without sample volume conservation. Smart. Mater. Struct. 2015, 24, 05509.

[28] Vudayagiri, S.; Junker, M. D.; Skov, A. L. Factors affecting the surface and release properties of thin polydimethylsiloxane films. Polymer journal, 2013, 45, 871-878 
[29] Vudayagiri, S.; Zakaria, S.; Yu, L.; Hassouneh, S. S.; Benslimane, M.; Skov, A.

L. High breakdown-strength composites from liquid silicone rubbers. Smart. Mater.

Struct. 2014, 23, 105017.

[30] Jensen, M. K.; Bach, A.; Hassager, O.; Skov, A. L. Linear rheology of crosslinked polypropylene oxide as a pressure sensitive adhesive. Int. J. Adhes. Adhes. 2009, 29, 687-693. 
Table 1 Weibull parameters for the three types of samples

\begin{tabular}{|l|l|l|l|}
\hline & Single films & Single, plasma-treated films & Laminates \\
\hline$\eta[$ V/um $]$ & 129 & 122 & 121 \\
\hline$\beta$ & 39 & 41 & 27 \\
\hline$r^{2}$ & 0,97 & 0,92 & 0,94 \\
\hline
\end{tabular}


Figure 1 Overview of the plasma treatment process, followed by the layer to layer adhesion of the two PDMS layers.

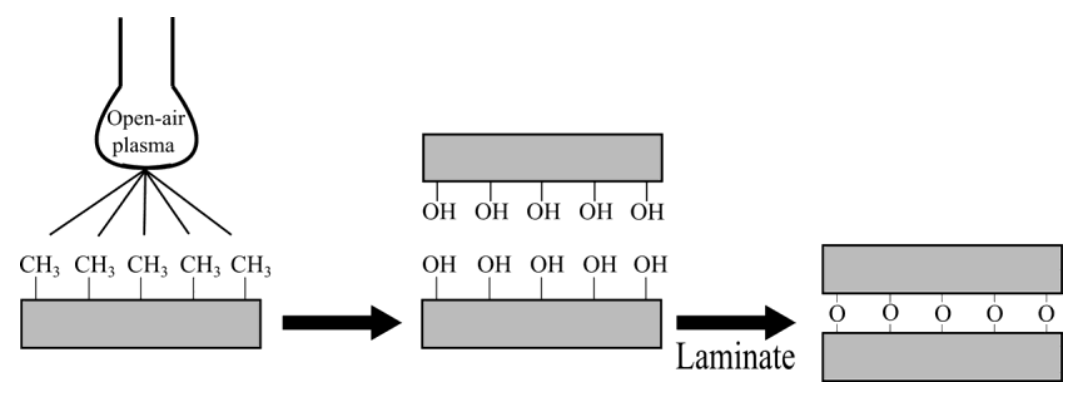


Figure 2 Linear rheological data of a laminate compared to a single film of LR3043/30 at $23{ }^{\circ} \mathrm{C}$. The thickness of the single film is $0.5 \mathrm{~mm}$ and $1 \mathrm{~mm}$ for the laminate.

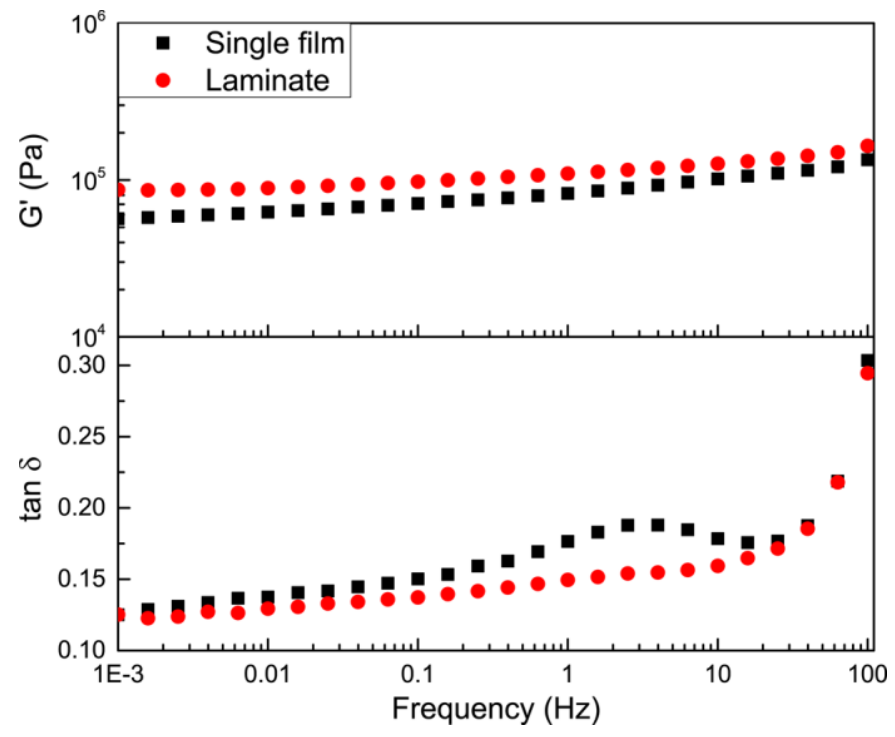


Figure 3 Breakdown strength for LR3043/30 laminates, where the plasma treatment is performed at different distances to the nozzle and speed. The single films have a thickness of $40 \mu \mathrm{m}$ and the laminate a thickness of $80 \mu \mathrm{m}$. The breakdown strengths illustrated are an average of 12 measurements.

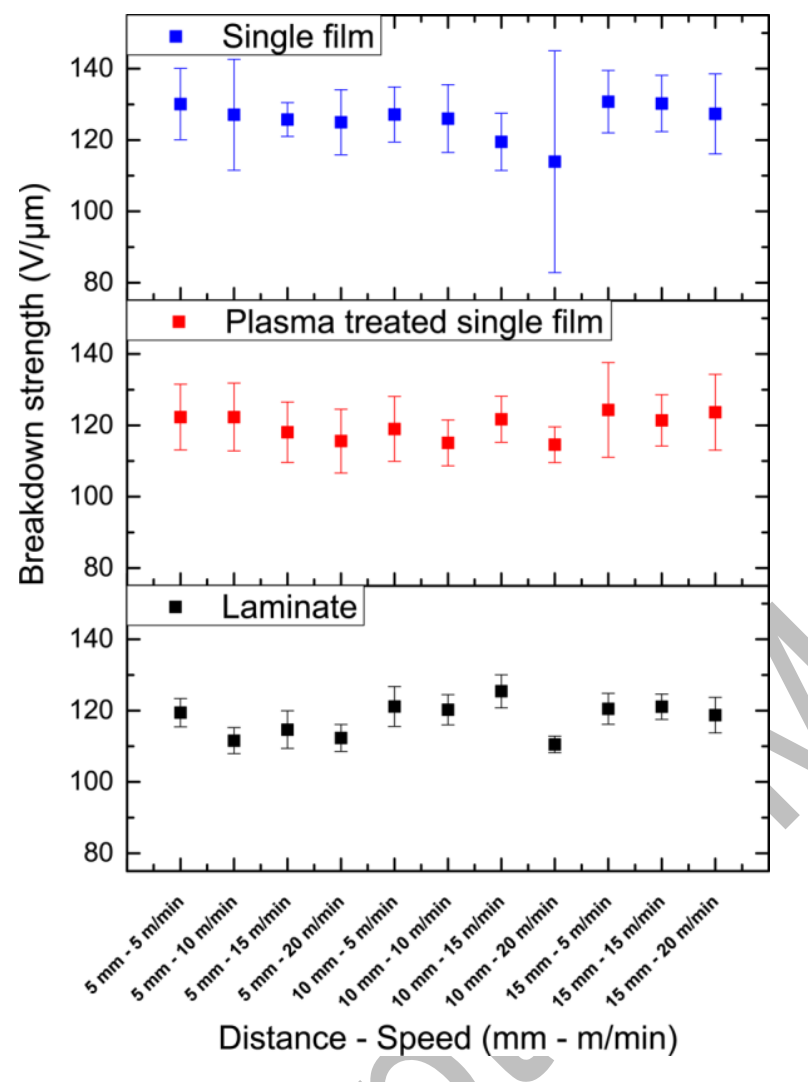


Figure 4 Peel forces of LR3043/30 laminates at $23{ }^{\circ} \mathrm{C}$. Typical standard deviations are $+/$ $10 \%$ since peel strengths are thickness dependent and the reading of peel strength from the plots is subjected to some error.

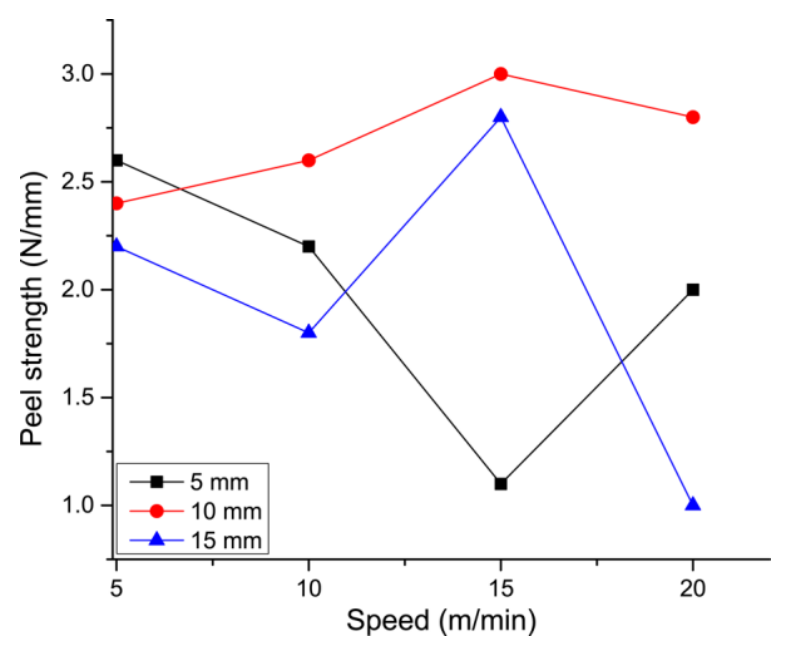

